\title{
A remote keyboard switch for the IBM PC/AT and compatibles
}

\author{
TIMOTHY HUNTER and J. ALEXANDER DALE \\ Allegheny College, Meadville, Pennsylvania
}

\begin{abstract}
A relatively simple, remote keyboard switch for the IBM PC/AT and compatibles is discussed. It may be used to isolate a subject from an experimenter when the experimenter is controlling an experiment with the PC. The device, based on a $4066 \mathrm{CMOS}$, when used in conjunction with a monochrome monitor and a color monitor, enables the experimenter to switch, manually or programmatically (TTL), between the local monitor and the local keyboard and a remote monitor and a remote keyboard for presentation of stimuli and for recording of subject's responses.
\end{abstract}

For many applications in psychology and other fields, it is desirable to separate the experimenter and the subject to preclude the subject's being distracted by the presence of another person (Cacioppo, 1989; Dale, Hudak, \& Wasikowski, 1991; Fridlund, 1989). Unfortunately, the personal computer is not designed to have two people interact through keyboards and monitors in different rooms. Although a local area network can adequately solve this problem, a less expensive solution would be to switch monitors and keyboards through a software program that would activate the appropriate keyboard and monitor via a remote keyboard switch. The IBM PC/AT can adequately switch between a monochrome and a color monitor, but it has no provision for switching between keyboards. The present remote keyboard switch was tested with several 80386/486based compatibles and should work with any compatible that takes an AT-style keyboard.

\section{Operation}

The switch input is a TTL level input, which allows a program running on the $\mathrm{PC}$ (in this case with a TecMar LabTender interface) to control which keyboard the computer will accept data from. The input can be driven from any TTL output ranging from a bit on the parallel port of the PC to any data acquisition and control card with a TTL level output.

A logic $1(+5 \mathrm{~V})$ on the switch input selects the remote keyboard, while logic $0(0 \mathrm{~V})$ or no input selects the local keyboard. Switch S1 manually selects the remote keyboard for testing or if automatic program control is not needed. LED D1 is lit when the remote keyboard is selected.

\section{Circuit Description}

As can be seen in Figure 1, data are sent to and from the keyboard in a serial format on pin 2 of the keyboard connector (usually located at the rear of the computer), which is labeled KeyData; these data are clocked by a sig-

Correspondence should be addressed to J. A. Dale, Allegheny College, Meadville, PA 16335. nal on pin 1 of the same connector, appropriately labeled KeyClk. By controlling which keyboard's KeyData and KeyClk lines are connected to the PC, we can control which keyboard it accepts data from. This is accomplished by a 4066 CMOS (see parts list) quad analog switch designated U1. A logic 1 on the control pin of an individual switch will allow signals to pass bidirectionally through the switch. A logic 0 opens the switch.

The local keyboard's KeyData and KeyClk lines are connected to U1c pin 4 and Ula pin 1, respectively; the control inputs pin 5 and pin 13 of U1c and Ula are connected together and to the output (pin 2) of U2a, a CMOS inverter. The remote keyboard's KeyData and KeyClk lines are connected to U1d pin 8 and U1b pin 11, respectively, the control inputs pin 6 and pin 12 of U1d and U1b are also connected together and to the input (pin 1) of U2a. The PC's KeyClk line is connected to Ula pin 2 and Ulb pin 10; the KeyData line is then connected to Ulc pin 3 and U1d pin 9. This forms a simple double-pole doublethrow switch. Since the control inputs of each pair of switches are connected together by an inverter, only one pair will be "on" at a time. The inverter input is connected to the remote keyboard switch's switch input. S1 manually switches the switch input between logic 1 and logic 0 through R1. R4 prevents any damage to the external control device (if connected) when $\mathrm{S} 1$ is closed. Q1 is biased through R3 from the remote keyboard switch's switch input also and when the input is logic 1 , Q1 turns on lighting LED D1. R2 limits the current flow through D1. Resistors R5, R6, R7, and R9 are used to pull the deselected keyboard's KeyData and KeyClk lines low (logic 0 ), thereby inhibiting it from buffering any keypresses. Power for the circuit is obtained from the keyboard power supply lines on pins 4 and 5 of the keyboard cable. CMOS chips are used to keep the current consumption minimal. All unused inputs of U2, a 4069 CMOS hex inverter, are tied to ground.

\section{Construction}

Since the circuit is relatively simple and has a low parts count, point-to-point wiring or wire-wrapping techniques 


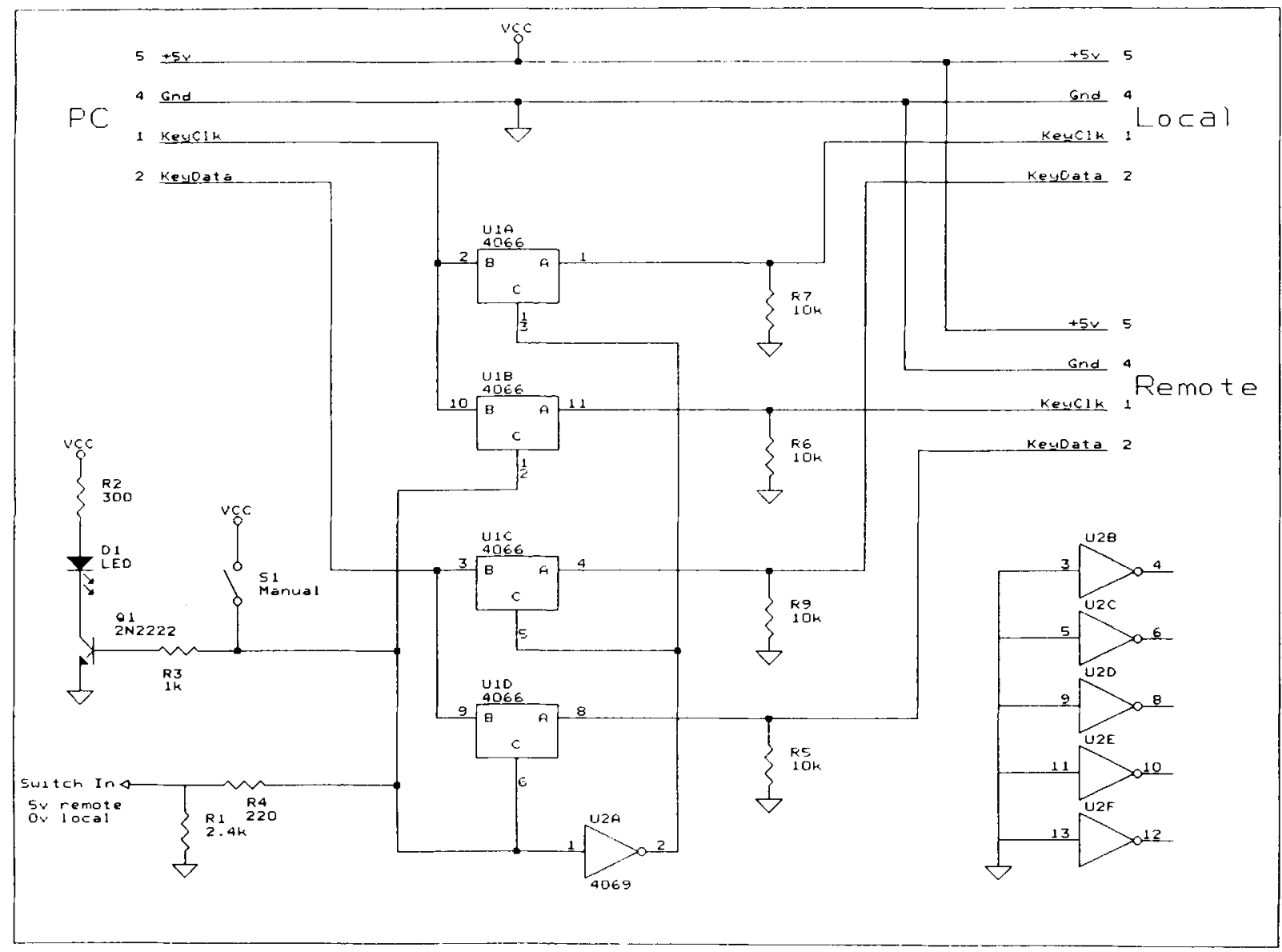

Figure 1. Schematic diagram of remote keyboard switch.

can be used to construct the project. In our case, we used point-to-point wiring on a small piece of $0.1 \times 0.1$ perf board. Keyboard connections were made by soldering inline DIN connectors to $1-\mathrm{m}$-long pieces of 6 conductor stranded cable and then soldering the other ends directly to the perf board. Mounting the board in a small utility box provides strain relief for the cables and a convenient place to mount the switch and LED. The control switch connection was made by mounting a binding post on the utility box as well.

\section{Discussion}

The present remote keyboard switch allows the experimenter to switch either manually or by using software from one keyboard to another. With the present $A / D$ board, the BASIC line, "Out 828,4," switches the keyboards to activate the remote keyboard through a TTL +5 -V output from the LabTender to the remote keyboard switch. The BASIC line "Out 828,0" causes the switch to reactivate the local keyboard. Additionally, the device clears the keyboard upon switching so that haphazard data entered previously will not interfere with on-line data collection.

With this device, a program written in QuickBASIC, a color monitor, and a monochrome monitor, we have been able to use an IBM PC/AT computer in an experimental control room to control timing and displays and to monitor keyboard responses in a separate subject room. As well, we have been able to monitor physiological measures simultaneously through the other channels of the A/D board mentioned above (see Emanuele, Dale, \& Klions, 1990; Stickle, Dale, \& Klions, 1991).

\section{REFERENCES}

CACIOPpo, J. (1989) Psychophysiological investigations of social processes: Physiological effect of human presence. Psychophysiology, 26, 10.

Dale, J. A., Hudak, M. A., \& Wasikowski, P. (1991). Effects of dyadic participation and awareness of being monitored on facial action during exposure to humor. Perceptual \& Motor Skills, 73, 984-986.

Emanuele, S., Dale, J. A., Klions, H. L. (1990). Problem solving versus humor on a computer as a function of computer anxiety: Facial action, zygomatic EMG and skin conductance level. Psychophysiology, 27, $\mathbf{5 2 8 .}$

Frudlund, A. J. (1989). Social functions of implicit smiling: Potentiation by an implicit audience. Psychophysiology, 26, 27.

Stickle, D., DAle, J. A., KLIONS, H. L. (1991). Social anxiety as a self-handicapping strategy: Facial action, corrugator and zygomatic EMG, Palmer SCL. Psychophysiology, 28, S52. 


\begin{tabular}{|c|c|c|c|c|}
\hline \multicolumn{5}{|c|}{$\begin{array}{c}\text { APPENDIX } \\
\text { Parts List } \\
\end{array}$} \\
\hline Quantity & $\begin{array}{c}\text { Fig. } 1 \\
\text { Code }\end{array}$ & Description & Part No. & Source \\
\hline 1 & D1 & LED & P367 & DigiKey \\
\hline 1 & Q1 & 2N2222 NPN general purpose & PN2222 & DigiKey \\
\hline 1 & $\mathrm{R} 1$ & $2.4-\Omega .5-W$ resistor & $2.4 \mathrm{KH}$ & DigiKey \\
\hline 1 & R2 & $300-\Omega .5-W$ resistor & $300 \mathrm{H}$ & DigiKey \\
\hline 1 & R3 & $1 \mathrm{k}-\Omega .5-\mathrm{W}$ resistor & $1 \mathrm{KH}$ & DigiKey \\
\hline 1 & R4 & $220-\Omega .5-W$ resistor & $220 \mathrm{H}$ & DigiKey \\
\hline 4 & $\begin{array}{l}\text { R5, R6 } \\
\text { R7, R9 }\end{array}$ & $10 \mathrm{k}-\Omega .5-\mathrm{W}$ resistor & $10 \mathrm{KH}$ & DigiKey \\
\hline 1 & S1 & SPST toggle switch & CKN1000-ND & DigiKey \\
\hline 1 & U1 & 4066 CMOS IC & CD4066BE & DigiKey \\
\hline 1 & $\mathrm{U} 2$ & 4069 CMOS IC & CD4069BE & DigiKey \\
\hline 1 & & keyboard connectors male & D-5P & Altex \\
\hline 2 & & keyboard connectors female & D-5S & Altex \\
\hline 2 meters & & 6 conductor stranded cable & 4306 & Altex \\
\hline 1 & & utility box & $704 \mathrm{~K}-\mathrm{ND}$ & DigiKey \\
\hline & & cover & 2045K-ND & DigiKey \\
\hline 1 & & perf board & $3405 \mathrm{~K}-\mathrm{ND}$ & DigiKey \\
\hline 1 & & binding post & $\mathrm{J} 370$ & DigiKey \\
\hline 2 & & IC sockets solder & C8914 & DigiKey \\
\hline & & IC sockets wire wrap & C8114 & DigiKey \\
\hline
\end{tabular}

(Manuscript received June 23, 1992;

revision accepted for publication September 30, 1992.) 